\title{
Determination of the Macroscopic Elastic Constants of A Phase Embedded In A Multiphase Polycrystal -Application to the $\beta$-Phase of A Ti-17 Titanium Based Alloy
}

\author{
S. Fréour ${ }^{1}$, D. Gloaguen ${ }^{1}$, M. François ${ }^{2}$, R. Guillén ${ }^{1}$, E. Girard ${ }^{1}$ and J. Bouillo ${ }^{3}$ \\ ${ }^{1}$ Laboratoire d'Applications des Matériaux à la Mécanique (LAMM)
}

L.A.M.M. - C.R.T.T. - Boulevard de l'Université - BP 406 - 44602 Saint Nazaire - France

Tel. : +33 02.40.17.26.20 Fax : +33 02.40.17.26.18. E mail : freour@lamm.univ-nantes.fr

${ }^{2}$ Laboratoire des Systèmes Mécaniques et d'Ingénierie Simultanée (LASMIS)

Univ. De Technologie de Troyes - 12 Rue Marie Curie - BP 2060 - 10010 Troyes - France

${ }^{3}$ Département Génie Civil - IUT de Saint Nazaire - BP 420 - 44606 Saint Nazaire - France

Keywords: two-phases materials, Ti-17, residual stresses, self-consistent models, elastic constants.

\begin{abstract}
A one-site elastic self-consistent model following the mathematical formalism introduced by Kröner and Eshelby (KE) has been developed in order to solve the case of multiphase materials. This model has been applied to duplex steels and aluminium - silicium carbide Metal Matrix Composites (MMC) in the aim to study the evolution of their stiffness at pseudomacroscopic scale. Simulations justify the usually implicit hypothesis of the identity of the elastic moduli of a given phase, at macroscopic and pseudomacroscopic scales. The implementation of KE model by this hypothesis yields a new implicit formulation for the stiffness of a given unknown phase embedded in a two-phases material. This original characterization method will be applied to the $\beta$-phase of Ti17 alloy. The singular behaviour in terms of residual pseudomacrostress of each phase after uniaxial loadings will be deduced from these data.
\end{abstract}

\section{Introduction}

Elasticity constants, Young modulus and Poisson ratio constitute fundamental data in residual stress analysis. They are especially essential to determine stresses from measured strains through diffraction means. Nevertheless, direct characterization methods of macroscopic elastic moduli should generally be applied to single-phase samples. It explains why the elastic constants of phases stable at high temperature of numerous two-phases materials are often not well known. $\alpha+\beta$ titanium or zirconium alloys illustrate this property [1]. It will be demonstrated in the following that the combination of ultrasonic and mechanic tests to micromechanical self consistent simulation models provides the calculation of these constants under precise but justified conditions.

\section{1 - Multi-phase one site self consistent elastic scheme}

1 - 1 Study of pseudomacroscopic stiffness in multi-phase materials. In order to solve the case of multi-phase materials, one will easily extend the fundamental hypothesis of Kröner and Eshelby single-phase self consistent model [2]. Let us consider a given $\alpha$-phase embedded in a multi-phase polycrystal. It has been demonstrated in [3] that the pseudomacroscopic stiffness tensor $\mathrm{C}^{\alpha}$ of this phase was given by the relation :

$\mathrm{C}^{\alpha}=\left\langle\mathrm{c}^{\alpha}(\Omega) \cdot \mathrm{A}(\Omega)\right\rangle_{\alpha} \cdot\langle\mathrm{A}(\Omega)\rangle_{\alpha}^{-1}$ 
Where $\mathrm{c}^{\alpha}(\Omega)$ denotes $\alpha$ phase single crystal elastic stiffness referred to a sample fixed coordinate system whose orientation is given by $\Omega$. A $(\Omega)$ symbolize the fourth order strain localization tensor expressed by :

$$
\mathrm{A}(\Omega)=\left[\mathrm{E} . .\left\{\mathrm{c}^{\alpha}(\Omega)-\mathrm{C}\right\}+\mathrm{I}\right]^{-1}
$$

I is the identity tensor, $\mathrm{C}$ the elastic modulus of the homogeneous equivalent multi-phase polycrystalline medium. E denotes Hill's tensor which depends on $\mathrm{C}$ value and on the morphology assumed for the crystallites [4]. One could express $\mathrm{C}$ as an average on each phase $\mathrm{i}$ of the material :

$\mathrm{C}=\left\langle\mathrm{C}^{\mathrm{i}} \cdot .\left[\mathrm{E} . .\left(\mathrm{C}^{\mathrm{i}}-\mathrm{C}\right)+\mathrm{I}\right]^{-1}\right\rangle$

$\alpha$-phase pseudomacroscopic stiffness is quite different from the expression obtained in the singlephase material, which could be obtained assuming that $\alpha$ grains are embedded in a homogeneous equivalent medium with stiffness equals to $C^{\alpha}$ instead of $C$ in (Eq. 1-2) :

$$
\mathrm{C}^{\alpha}(\text { single - phase })=\left\langle\mathrm{c}^{\alpha}(\Omega) \cdot\left[\mathrm{E} .\left\{\left[\mathrm{c}^{\alpha}(\Omega)-\mathrm{C}^{\alpha}\right\}+\mathrm{I}\right]^{-1}\right\rangle_{\alpha}=\left\langle\mathrm{c}^{\alpha}(\Omega) \cdot \mathrm{A}(\Omega)\right\rangle_{\alpha}\right.
$$

Differences observed between (Eq.1) and (Eq. 4) are explained by the influence on $C^{\alpha}$ of the other phases elastic properties, through the value of $\mathrm{C}$ and thus, of $\mathrm{E}$. The multiplier term $\langle\mathrm{A}(\Omega)\rangle_{\alpha}^{-1}=\left\langle\left[\mathrm{E} . .\left\{\mathrm{c}^{\alpha}(\Omega)-\mathrm{C}\right\}+\mathrm{I}\right]^{-1}\right\rangle_{\alpha}^{-1}$ might be considered as a deviation factor corresponding to the average interaction between $\alpha$-crystallites and the infinite equivalent medium. In consequence, in a multi-phase material, the nature and the proportion of the other phases forming the polycrystal should affects the average elastic properties of a given $\alpha$-phase. As a result, the pseudomacrostiffness of a given phase might not be identified to its macroscopic stiffness, when it is integrated in a n-phases material.

\section{1-2 Justification of the assumption of stiffness identity for a phase at macroscopic and pseudo} macroscopic scales. Numerous simulations have been done in the aim to quantify two-phases effects on pseudomacroscopic stiffness. Let us consider the results obtained in the cases of two usually encountered materials presenting interesting properties (cf Table 1):

- duplex steels, whose two phases are constituted by strongly anisotropic single crystals but present similar macroscopic elastic behaviours.

- Aluminium - silicon carbide MMC, present strong elastic pseudomacroscopic heterogeneities and one phase whose single crystal is quasi-isotropic (aluminium).

Although pseudo-macro-stiffness varies with the nature and the volume fraction of the other phase, the bulk modulus $\mathrm{K}$ of each phase remains perfectly constant at pseudomacroscopic scale. It is well known that $\mathrm{K}$ values are, since it is an invariant of the stiffness tensor, identical in single crystal (mesoscopic scale) and in cubic structure single phase polycrystals (macroscopic scale). In consequence a variation of the bulk modulus would not be physically acceptable. Results obtained satisfy this criterion. This confirms the numerical validity of the model developed which implies the deviation of pseudo macro stiffness values in order to assure the consistence criterion.

Table 1 demonstrates that pseudo macro stiffness components deviation due to secondary phase interactions remains weak and, in most case, negligible. Two-phase influence is particularly weak, when the single crystal of the phase considered is almost elastically isotropic (Al), or when the two phases have similar macroscopic mechanical properties (duplex steels). 
In consequence, this study justifies the usually implicit hypothesis of the identity of the stiffness of a given phase, at macroscopic or pseudomacroscopic scale, whatever the nature of the polycrystalline aggregate in which it is embedded.

Table 1: Two-phase influence on pseudomacrostiffness values [3]

\begin{tabular}{|l|r|r|r|r|r|}
\hline Pseudo-macro stiffness tensor components and bulk moduli calculated in the case of Al-SiC MMC \\
\hline $\mathrm{f}(\mathrm{Al})$ & -0.00 & 0.25 & 0.50 & 0.75 & $\sim 1.00$ \\
\hline $\mathrm{C}_{11}(\mathrm{Al})[\mathrm{GPa}]$ & 112.2 & 112.2 & 112.1 & 112.1 & 112.1 \\
\hline $\mathrm{C}_{12}(\mathrm{Al})[\mathrm{GPa}]$ & 59.3 & 59.3 & 59.3 & 59.3 & 59.4 \\
\hline $\mathrm{K}(\mathrm{Al})[\mathrm{GPa}]$ & 76.9 & 76.9 & 76.9 & 76.9 & 77.0 \\
\hline $\mathrm{C}_{11}(\mathrm{SiC})[\mathrm{GPa}]$ & 437.7 & 434.2 & 430.8 & 428.1 & 426.7 \\
\hline $\mathrm{C}_{12}(\mathrm{SiC})[\mathrm{GPa}]$ & 97.6 & 99.4 & 101.1 & 102.5 & 103.2 \\
\hline $\mathrm{K}(\mathrm{SiC})[\mathrm{GPa}]$ & 211.0 & 211.0 & 211.0 & 211.0 & 211.0 \\
\hline Pseudo-macro stiffness tensor components and bulk moduli calculated in the case of Al-SiC MMC \\
\hline $\mathrm{f}\left(\mathrm{Fe}_{\alpha}\right)$ & -0.00 & 0.25 & 0.50 & 0.75 & $\sim 1.00$ \\
\hline $\mathrm{C}_{11}\left(\mathrm{Fe}_{\alpha}\right)[\mathrm{GPa}]$ & 281.0 & 281.1 & 281.2 & 281.2 & 281.2 \\
\hline $\mathrm{C}_{12}\left(\mathrm{Fe}_{\alpha}\right)[\mathrm{GPa}]$ & 112.9 & 112.8 & 112.8 & 112.8 & 112.7 \\
\hline $\mathrm{K}\left(\mathrm{Fe}_{\alpha}\right)[\mathrm{GPa}]$ & 168.9 & 168.9 & 168.9 & 168.9 & 168.9 \\
\hline $\mathrm{C}_{11}\left(\mathrm{Fe}_{\gamma}\right)[\mathrm{GPa}]$ & 250.6 & 250.8 & 251.1 & 251.3 & 251.5 \\
\hline $\mathrm{C}_{12}\left(\mathrm{Fe}_{\gamma}\right)[\mathrm{GPa}]$ & 97.9 & 97.8 & 97.7 & 97.6 & 97.5 \\
\hline $\mathrm{K}\left(\mathrm{Fe}_{\gamma}\right)[\mathrm{GPa}]$ & 148.8 & 148.8 & 148.8 & 148.8 & 148.8 \\
\hline
\end{tabular}

\section{2 - Determination of the elastic macroscopic constants of a phase embedded in a multi-phase material}

2 - 1 Kröner and Eshelby model implementation. Let us introduce the hypothesis justified in the previous paragraph in the formalism of the self consistent model. One would demonstrate that this assumption yields a new expression of the elastic properties of an unknown phase embedded in a multiphase material. In fact, the stiffness tensor of the studied phase will be given as a function of the elastic constants of the polycrystal and of the other phases. Now, let us assume that the macroscopic elastic constants of a n-phases polycrystal are known, just as those of n- 1 phases. For example, the phases $\{1,2, \ldots, i, \ldots, n-1\}$ whose volume fractions are respectively denoted by $\left\{f^{1}\right.$, $\left.\mathrm{f}^{2}, \ldots, \mathrm{f}^{\mathrm{i}}, \ldots, \mathrm{f}^{\mathrm{n}-1}\right\}$. The implicit equation (Eq. 3) may be developed as a $\mathrm{n}$ terms summation :

$$
C=f^{n} C^{n} . .\left[E . .\left(C^{n}-C\right)+I\right]^{-1}+\sum_{i=1}^{n-1} f^{i} C^{i} . .\left[E . .\left(C^{i}-C\right)+I\right]^{-1}
$$

The stiffness tensor of the unknown phase denoted by $n$ verifies another implicit equation :

$$
\mathbf{C}^{\mathbf{n}}=\frac{1}{\mathbf{f}^{\mathbf{n}}} \mathbf{C} . .\left[E . .\left(\mathbf{C}^{\mathbf{n}}-\mathbf{C}\right)+\mathbf{I}\right]-\sum_{\mathbf{i}=\mathbf{1}}^{\mathbf{n}-1} \frac{\mathbf{f}^{\mathbf{i}}}{\mathbf{n}^{\mathbf{n}}} \mathbf{C}^{\mathbf{i}} . .\left[E . .\left(\mathbf{C}^{\mathbf{i}}-\mathbf{C}\right)+\mathbf{I}\right]^{-1} . .\left[E . .\left(\mathbf{C}^{\mathbf{n}}-\mathbf{C}\right)+\mathbf{I}\right]
$$

In the point of view of $\mathrm{KE}$ model, this assumption leads to represent a $\mathrm{n}$-phases material under the form of $\mathrm{n}$ single phase inclusions embedded in an infinite homogeneous medium. The elastic properties of the matrix are the same as those of the multi-phase polycrystal and satisfy the equation (Eq. 5) in which one will assume the elastic constants of a given phase i identical to the stiffness of the pure single phase $\mathrm{i}$.

2 - 2 Application to the determination of Ti-17 $\beta$-phase macroscopic elastic constants. In the case of titanium alloys, the presence of alloying elements may provide the stabilization at room 
temperature of a certain volume fraction of the $\beta$ phase. It is difficult to obtain a single phase polycrystal of $\beta$ through thermo-mechanical treatments in order to characterize its elastic properties. Any variation in the fractions of the different alloying elements modifies the nature of the alloy and in consequence, the mechanical properties of the $\beta$-phase [5]. Thus, it is necessary to measure the elastic constants of the unknown phase directly on the $\alpha+\beta$ two-phases polycrystal. For the study of this kind of material, our model is based on the following equation deduced from (Eq. 6) :

$$
\mathbf{C}^{\beta}=\frac{1}{\mathbf{f}^{\beta}} \mathbf{C} . .\left[\mathbf{E} . .\left(\mathbf{C}^{\beta}-\mathbf{C}\right)+\mathbf{I}\right]-\frac{\mathbf{f}^{\alpha}}{\mathbf{f}^{\beta}} \mathbf{C}^{\alpha} \ldots\left[\mathbf{E} \ldots\left(\mathbf{C}^{\alpha}-\mathbf{C}\right)+\mathbf{I}\right]^{-1} \ldots\left[\mathbf{E} \ldots\left(\mathbf{C}^{\beta}-\mathbf{C}\right)+\mathbf{I}\right]
$$

The application of this equation implies that the pseudomacroscopic mechanical behaviour of $\alpha$ phase is perfectly determined. This behaviour will be identified to $\alpha$ single phase polycrystal elastic moduli. The macroscopic stiffness tensor $\mathrm{C}$ of the polycrystal is also needed. The corresponding information is contained in the Young modulus $\mathrm{Y}$ and the Poisson Ratio $v$ of the two-phase material. As a result, only these parameters should be precisely measured in the case considered.

This method has been applied in order to determine the macroscopic stiffness tensor of Ti-17 $\beta$ phase. This alloy is constituted by $70 \%$ in volume of hexagonal $\alpha$-phase and $30 \%$ of body centered cubic $\beta$-phase. $\alpha$-phase macroscopic elastic constants have been calculated from its single crystal stiffness (given in Table 2) through a single phase scale transition model according to (Eq. 4). The results are given in Table 3 .

Table 2 : $\alpha$-Ti single crystal elastic constants[6].

\begin{tabular}{|r|r|r|r|r|r|}
\hline $\mathrm{c}_{11}[\mathrm{GPa}]$ & $\mathrm{c}_{12}[\mathrm{GPa}]$ & $\mathrm{C}_{13}[\mathrm{GPa}]$ & $\mathrm{c}_{33}[\mathrm{GPa}]$ & $\mathrm{C}_{44}[\mathrm{GPa}]$ & $\mathrm{c}_{66}[\mathrm{GPa}]$ \\
\hline 162.4 & 92.0 & 69.0 & 180.7 & 46.7 & 35.2 \\
\hline
\end{tabular}

Table 3 : Ti-17 $\alpha$-phase macroscopic elastic constants calculated through KE model.

\begin{tabular}{|r|r|r|r|}
\hline $\mathrm{C}_{11}[\mathrm{GPa}]$ & $\mathrm{C}_{12}[\mathrm{GPa}]$ & $\mathrm{Y}[\mathrm{GPa}]$ & $\mathrm{v}$ \\
\hline 165.1 & 78.4 & 114.6 & 0.322 \\
\hline
\end{tabular}

Samples have been machined in order to perform to tensile and ultrasonic tests. The Young modulus of Ti-17 polycrystal has been determined from the slope of the tensile curve obtained. The ultrasonic longitudinal waves celerity $C_{L}$ depend on the elastic constants of the material as follow :

$$
C_{L}=\sqrt{\frac{Y(1-v)}{\rho(1+v)(1-2 v)}}
$$

Where $\rho$ represents the density of the material.

Its Poisson ratio satisfies the following equation obtained from eq. 8 :

$$
v=\frac{-C_{L}^{2} \rho+Y+\sqrt{9 C_{L}^{4} \rho^{2}-10 C_{L}^{2} \rho Y+Y^{2}}}{4 C_{L}^{2} \rho}
$$

Density, Young's modulus and ultrasonic longitudinal celerity of the Ti-17 have been precisely determined. The Poisson ratio has then been deduced :

$$
\rho=4644 \pm 7 \mathrm{~kg} / \mathrm{m}^{3} \quad \mathrm{Y}=110.0 \pm 2.2 \mathrm{GPa} \quad \mathrm{C}_{\mathrm{L}}=6040 \pm 11 \mathrm{~m} / \mathrm{s} \quad v=0.340 \pm 0.005
$$

Ti-17 density is quite similar to pure titanium density, which is announced to $4510 \mathrm{~kg} / \mathrm{m}^{3}$, according to [7]. The difference observed is obviously explained by the alloying elements contained in Ti-17. 
In the same way, weak discrepancy between the Young moduli of Ti-17 and $\alpha$-Ti (3.5\%) is due to the high volume fraction of $\alpha$-phase in Ti-17.

$C_{11}$ and $C_{12}$ components of the multi-phase polycrystal stiffness tensor could easily be deduced from $\mathrm{Y}$ and $\mathrm{v}$ :

$C_{11}=\frac{v Y-Y}{2 v^{2}+v-1} \quad C_{12}=-\frac{v Y}{2 v^{2}+v-1}$

The macroscopic elastic properties of the multi-phase $\mathrm{Ti}-17$ polycrystal and those of the Ti-17 $\beta$ phase (calculated from Eq. 7, 10) are summarized in Table 4.

Table 4 : macroscopic elastic constants of Ti-17 and Ti-17 $\beta$-phase polycrystal.

\begin{tabular}{|c|r|r|r|r|}
\hline \multirow{2}{*}{ Ti-17 polycrystal } & $\mathrm{C}_{11}[\mathrm{GPa}]$ & $\mathrm{C}_{12}[\mathrm{GPa}]$ & $\mathrm{Y}[\mathrm{GPa}]$ & $\mathrm{v}$ \\
\cline { 2 - 5 } & $169.4 \pm 4.2$ & $87.4 \pm 3.9$ & $110.0 \pm 1.6$ & $0.340 \pm 0.005$ \\
\hline \multirow{2}{*}{ Ti-17 $\beta$-phase } & $\mathrm{C}_{11}[\mathrm{GPa}]$ & $\mathrm{C}_{12}[\mathrm{GPa}]$ & $\mathrm{Y}[\mathrm{GPa}]$ & $\mathrm{v}$ \\
\cline { 2 - 5 } & $183.0 \pm 12.6$ & $111.0 \pm 13.0$ & $99.3 \pm 6.9$ & $0.377 \pm 0.015$ \\
\hline
\end{tabular}

From the experimental deviation on the constants measured in the alloy, the errors on $\beta$-phase Young modulus and Poisson ratio have been quantified. They have been respectively estimated to $7 \%$ and $4 \%$.

The elastic constants of $\alpha$ and $\beta$ phases in Ti-17 deviate from about $15 \%$. Thus, they present an elastic mechanical behaviour quite similar. In the point of view of the multi-phase influence, the case of Ti-17 could be compared to the case of duplex steels. This remark a posteriori justifies the assumption of stiffness identity at pseudomacroscopic and macroscopic scales for any phase of the material. The weak elastic anisotropy of $\alpha$-phase single-crystal is another argument justifying this hypothesis.

The analyze is based on the assumption of mechanical macroscopic and pseudomacroscopic isotropy. But, the method could be extended to the case of textured material if the ODF and the macroscopic elastic anisotropy are taken into account.

\section{3 - Simulation of residual pseudomacrostresses after tensile uniaxial loading}

A self consistent elastic-plastic model has been developed in the aim to simulate the average residual stresses undergone by each phase of Ti-17 alloy submitted to macroscropic loadings corresponding to small plastic strains. Figure 1 details the curves obtained. It shows that Ti-17 alloy exhibit a singular behaviour in terms of pseudomacroscopic residual stresses. Pseudomacrostress sign inversion constitutes an original property. In fact, $\alpha$-phase presents a Young modulus higher than $\beta$-phase modulus. Consequently, when the strain is purely elastic, the axial stresses are in tension for $\alpha$ phase and compressive for $\beta$ phase. However, as $\beta$ phase remains elastic at small plastic strains [8], it becomes harder than $\alpha$-phase during plastic deformation. Thus, axial residual pseudomacrostresses signs change during plastic deformation.

Usually, the softer phase presents the lower elasticity limit, that's why it keeps a tensile axial residual pseudomacrostress whatever the plastic strain level. At the opposite, the harder phase has often the higher elasticity limit, thus, it keeps a compression axial residual pseudomacrostress whatever the plastic strain rate. The case of pearlitic steels illustrates this classical property of twophases materials [9].

Figure 1 : Ti-17 macroscopic stress-strain curve and deviation between longitudinal pseudomacrostresses and macrostresses simulated at small plastic strain rates. 

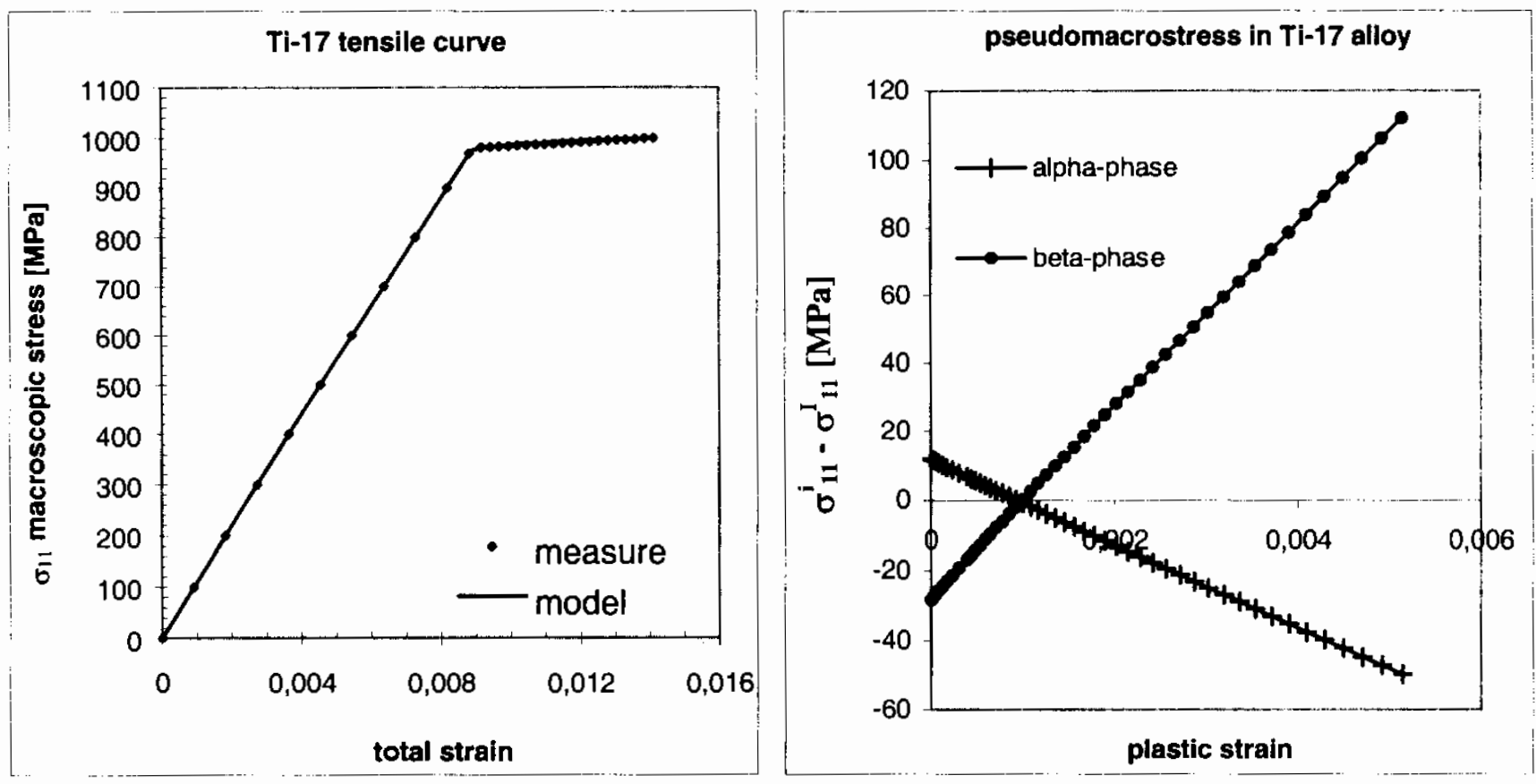

\section{Conclusion}

A three scales self consistent model has been developed in the aim to take into account the effects of the other phases on the pseudomacroscopic elastic moduli of a given phase. Numerous simulations have been done in particular in the cases of duplex steels and aluminium - silicon carbide MMC. The numerical results justify the assumption of the identity of the pseudomacrostiffness with the macroscopic stiffness of the pure single phase. The implementation of the self consistent model with this supplementary criterion yields a new implicit equation leading to the determination of the pseudomacroscopic stiffness of an unknown phase embedded in a multiphase material, from the elastic properties of the polycrystal and ot the other constituents. This original method has been applied to the characterization of Ti-17 $\beta$-phase macroscopic elastic properties. These data allowed to predict the average residual stresses in each phase of Ti-17 alloy. Moreover this study provides the values necessary to determine of Ti-17 $\beta$-phase X-Ray Elastic Constants and single crystal elastic constants.

\section{References :}

[1] D. T. Hawkins and R. Hultgren, «Constitution of binary alloys », Metals Handbook, vol 8, ASM, pp 251-376, 1973.

[2] E. Kröner, «Berechnung der elastichen Konstanten des Vielkristalls aus des Konstanten des Einkristalls », Z. Physik, 151, pp 504-518 (1958).

[3] S. Fréour, M. François and R. Guillén, «Multi phase effects on X-Ray Elasticity Constants", submitted to Acta Mat.

[4] J.D. Eshelby, "The determination of the Elastic Field of an Ellipsoidal Inclusion, and Related Problems, Proc. Of the Royal Society London, A241, pp 376-396, 1957.

[5] D. Kuroda, M. Niinomi, M. Morinaga, Y. Kato and T. Yashiro, « Design and mechanical properties of new $\beta$ type titanium alloys for implant materials ", Mater. Sci. Ing., A243, pp 244-249, 1998.

[6] G. Simmons and H. Wang, Cambridge, MA : M.I.T. Press, 1971.

[7] M. J. Donachie, « Titanium, a technical guide, second edition », ASM, 2000.

[8] X. Feaugas and M. Clavel, "Cyclic deformation behaviour of an $\alpha / \beta$ titanium alloy - I. Micromechanisms of plasticity under various loading paths », Acta mater., vol 45, 7, pp 2865-2701, 1997

[9] T. Hanabusa, H. Fujiwara and M. Nishida, « Residual microstress development in steels after tensile deformation », Proc of the ICRS 2, France, pp 555-560, 1988. 